\title{
Contralateral and heteromodal interaction effects in somatosensation: Do they exist?
}

\author{
WILLIAM R. UTTAL AND PAMELA SMITH
}

THE UNIVERSITY OF MICHIGAN

In this paper we concern ourselves with the problem of whether or not conditioning stimuli of other modalities or stimuli applied to a distant locus of the body interact with a sensitive test of somesthetic temporal acuity we have called the gap test. The results of the experiment indicate that visual stimuli do not exert an observable influence on this temporal judgment, but that auditory stimuli and contralateral stimulation in the same modality do show a significant but relatively small effect. This is compared and contrasted with the very large and persistent effect reported when the conditioning stimulus is applied to the same electrodes as the gap test. By comparing our results with experiments of similar design carried out on lower animats by other investigators, some insights into the neural localization of the reported effects is obtained.

The problem of heteromodal sensory interaction has been of recurrent interest to psychologists over the last few decades. More recently, clinical studies have stressed the masking effects of sound on the pain of dental drilling (Gardner \& Licklider, 1959) or have shown profound examples of what Bender (1952) calls "extinction." However, there appears to be very little direct experimental evidence that heteromodal inhibitory masking occurs within the somatosensory system. It is the purpose of this paper to consider the problem, using as our test probe an extremely sensitive measure of temporal acuity which we have called the gap test.

In an earlier paper (Uttal \& Krissoff, 1966) we explored the basic parameters of the psychophysical detection of gaps in a train of electrical pulses applied to peripheral nerves in the skin. Because of the unique stimulation situation-electrical stimuli produce compound action potentials in peripheral nerves on a one-to-one basis independent of somatosensory transducer action-these experiments speak directly to the problem of the neural code. Parameters of frequency, position, and numerosity were all shown to strongly affect the threshold for gap detection. Subsequently (Uttal \& Krissoff, 1967), we described a very strong and persistent refractory effect of a conditioning burst of similar stimuli applied to the same electrodes on the gap threshold.

In the present paper we consider the effects of other types of conditioning stimuli on the gap threshold. Considering the extreme variability of the threshold, when preceded by a conditioning stimulus, the gap detection test would, at first glance, seem to be a very appropriate test for cross sensory effects. The other types of conditioning stimuli used include bursts of electrical stimuli applied to the opposite side of the body and bursts of acoustic clicks or brief light flashes. The general results of the studies we shall report show the effect of these contralateral or heteromodal conditioning stimuli to be very small compared to the ipsilateral results of the previous experiment. These results provide a new insight into the problem of sensory interaction, a field which is currently particularly active in the neurophysiological literature. A number of both single and multiple cell recordings have shown that there is a wide variety of polysensory interactions produced by simultaneous stimulation of different sensory modalities when recording from associative rather than primary sensory areas. On the other hand, the compound evoked potentials recorded from the human scalp generally do not show such interaction.

\section{Subjects}

\section{METHOD}

The data presented in Fig. 2 are for five $\mathrm{Ss}$, in Fig. 3 for four Ss, in Fig. 4 for four Ss, and in Fig. 5 for five Ss. As in our earlier work, we emphasize that there is a considerable amount of inter- and intra-S variability and that the thresholds reported are sensitive to various factors affecting the vigilance of the Ss. All Ss, however, did exhibit the same general pattern of response. Differences in absolute levels are not considered particularly significant. Rather, relative levels constitute the data on which our conclusions are based. Experiments currently under way with forced choice psychophysical procedures do show, as expected, significantly lower absolute levels. In each of the experiments described here, a reference level is established for the situation in which no conditioning burst of any kind precedes the gap test, and it is with regard to this reference that we have drawn our conclusions.

\section{Procedure}

The main goal of the experiment was to determine if there was any effect of three different conditioning stimuli on the threshold for gap detection. Four different experiments were carried out. The first three replicated the experimental paradigm used in the study of ipsilateral masking (Uttal \& Krissoff, 1967). In these three experiments the conditioning burst was not a preceding train of stimuli applied 
to the same electrodes as the gap test but was a similar train applied to the other hand, a burst of light flashes or a burst of auditory clicks, respectively. Since our earlier experiment had shown significant effects caused both by the number of the stimuli in the conditioning train and the interval between the conditioning train and the gap test, both parameters were varied in these three experiments. The number of pulses in the conditioning burst (n) is not always equal to eight as shown in Fig. 1, but varied from block to block and might contain 1,4 , or 8 pulses. Since pilot studies indicated that any effect, if present, would be small, I, the interval between the conditioning train and the gap test, was not designed to vary as greatly as in the preceding experiment. Intervals between the conditioning burst and the gap test of 100,300 , and $500 \mathrm{msec}$ only were sampled.

Because of the very small effects observed in these three experiments, a fourth experiment was also carried out. In this experiment the burst of contralateral electrical, photic or acoustic stimuli started at the same time the pulses of the gap test began, rather than preceding it as in the first three experiments. The train of conditioning stimuli in this case was always $200 \mathrm{msec}$ long and the individual pulses were separated by equal intervals of $16 \mathrm{msec}$. The complete overlap of this conditioning burst and the gap test, and the large number of pulses, would be expected to accentuate any lesser effect which we might have missed with the long delays of the other three expeximents. In this experiment, each of the three conditioning situations was presented twice in each session along with two blocks without any conditioning burst.

Trials were presented in blocks of 75 in all experiments. Each block was constant in the number of pulses in the conditioning train, the modality of stimulation, and the interval between the conditioning train and the gap test. Within each block the precise sequence of stimuli is not definable since the psychophysical method used was a contingent one in which subsequent stimuli were selected on the basis of previous responses. The computer controlling the experiment did, however, print out a running protocol of gap size and the S's responses. The initial gap presented each day was $100 \mathrm{msec}$, well above the gap threshold. The S's responses forced the gap size down into the region of the threshold. In general, the flrst nine trials of each block were not included in the final average to avoid any artifact of the initial gap size. Step size was automatically varied, being set equal to $1 / 10$ of the range of responses on the previous block. In all cases the S's task remained the same-to report whether or not there was a gap or discontinuity in the otherwise regular train of impulses making up the gap test.

Each of the four experiments was run separately.
The 10 conditions for the first three experiments were presented in a constrained random order each day for 10 sessions for each $S$. The elght conditions of the fourth experiment were presented in a similarly constrained random order for five sessions. Data from all Ss were pooled. Therefore, each point on Figs. 2, 3, and 4 indicates the average of over 2000 estimates by all Ss. For Fig. 5 each point represents the average of 1650 estimates by all Ss.

\section{Apparatus}

The stimulus for the gap test is composed of a train of electrical pulse stimuli applied to the S's index and middle fingers of the left hand which were inserted into a pair of test tubes containing a body normal saline solution. Individual pulses in the train were constant current electrical pulses $.5 \mathrm{msec}$ in duration and with an amplitude of $4 \mathrm{~mA}$. In the first experiment in which electrical pulses were applied to the other side of the body, the $S$ inserted the index and middle fingers of his right hand into a similar pair of test tubes and received a conditioning train of electrical pulses. The $S$ responded either by pressing foot pedals or by means of a two-position manual switch to indicate whether he had or had not felt the gap.

The parameters of the gap test and the conditioning train are shown in Fig. 1. The stimulus pulses in both the conditioning train and the gap test were separated from each other by $16 \mathrm{msec}$, except for 1 , the interval between the conditioning train and the gap test and the gap itself. In the second experiment, the conditioning was a series of light flashes from a General Radio Corporation Strobotac placed about $3 \mathrm{ft}$ in front of the S's face but outside the acoustic cubicle in which he was sitting. The $S$ could not hear the clicks made by the Strobotac. In the third experiment, the conditioning train was a series of free-field acoustic clicks delivered through a loudspeaker placed $1 \mathrm{ft}$ in back of his head. In each case the various stimuli were timed as shown on the horizontal coordinate of their respective graphs. We have already described the psychophysical technique in an earlier paper (Uttal \& Krissoff, 1966). Briefly,

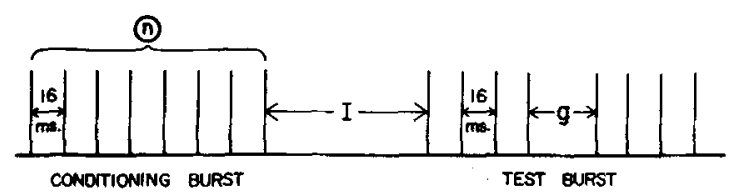

Fig. 1. A sample stimulus configuration. The conditioning burst might contain different numbers of pulses than that shown and might be made up of different forms of physical energy as described in the text. I defines the interval between the conditioning burst and the test burst and, along with $n$, the number of pulses, and the modality of the stimulus constitutes the independent variables manipulated in this experiment. The mean just detectable gap size (g) is in all cases the dependent variable. 


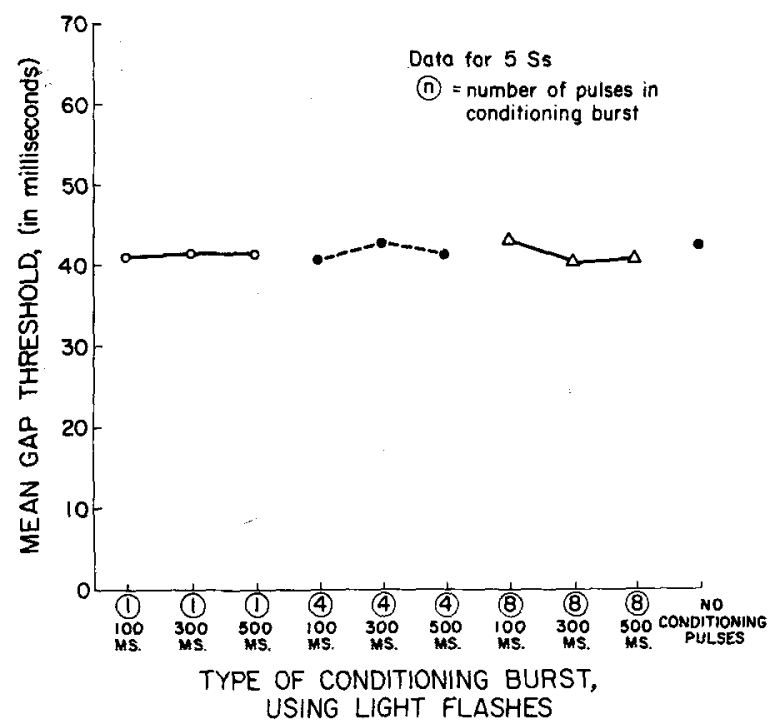

Fig. 2. The data for the experimental trials in which the conditioning burst is made up of a train of light flashes. The data for nine experimental conditions are shown, in which the number of pulses and the interval between the conditioning and test burst are both varied. The tenth point is a control to establish a reference level when there is no conditioning burst.

the entire experimental procedure was controlled by a small digital computer which presented the experimental trials according to a contingent up-anddown method of limits.

\section{RESULTS}

The results of the four experiments are plotted in Figs. 2, 3, 4, and 5. Figure 4 shows the results for the experiment in which the conditioning train was made up of a series of electrical pulses to the opposite hand. Figure 2 shows the results for the conditions in which the conditioning train was made up of a series of light flashes. Figure 3 presents the results for the situation in which the conditioning train was made up of auditory clicks. Figure 5 presents the results for the overlapping conditioning train.

Figure 4, unlike the other two figures, does show a slight effect for the cases in which $I=100 \mathrm{msec}$, but compared to Fig. 6 , it is seen to be a considerably smaller effect than that found for conditioning of the same hand. No effect at all can be observed in Figs. 2 and 3. Figure 5 presents the results for the overlapping train conditoning of Experiment 4. There appears to be little interaction produced by visual stimuli. However, contralateral stimulation with electrical pulses and acoustic conditioning appear to produce an inhibitory interaction, though considerably smaller than that produced by a conditioning stimulus applied $100 \mathrm{msec}$ prior to the gap test on the same level. The minimal size of these effects should be compared to the very effective and persistent effects found in the situation in which the conditioning stimulus is applied to the same electrodes as the gap test. That data is shown in Fig. 6, a plot of the results from that situation as reported in the earlier publication. Data on this figure have been normalized as described in that report.

\section{DISCUSSION}

There are two closely related but separate aspects of the problem of heteromodal interaction with which our data is concerned. The first concerns the presence of somatosensory interaction as a psychological phenomenon: Does it or does it not exist? The second concerns the representation of this type of sensory experience by various recordable states of the nervous system.

The study of sensory interaction is one of the fields in which a great deal of uncertainty surrounds the very existence of the basic phenomenon under investigation. For example, Walker and Sawyer (1961), in replicating Ogilvie's (1956a, b) papers, found that what had appeared to be very strong interaction effects (of a fluttering sound on flicker fusion) was most certainly an artifact mediated by an indirect modulation of the size of the pupil. This sort of effect is considerably different from the type of sensory interaction with which electrophysiologists believe they are concerned. Novak (1965) lists other experiments which have shown contradictory results in this same problem area. Another situation in which sensory interaction has been reported is the phenomenon of audio analgesia (Gardner \& Licklider, 1959). Nevertheless, however effective the technique may be in

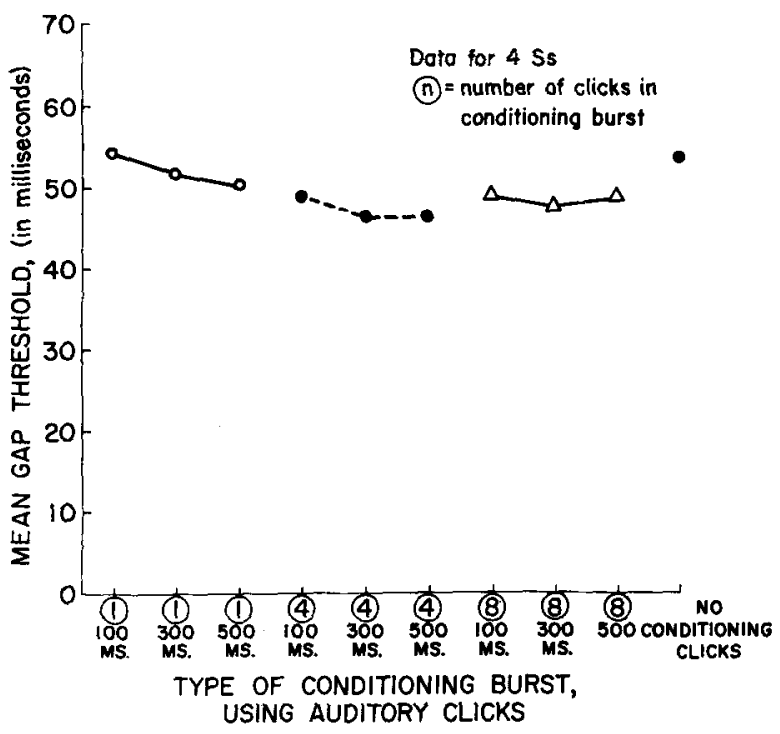

Fig. 3. The data for the experimental trials in which the conditioning burst is made up of auditory clicks. The arrangements of the data is the same as for Fig. 2. 


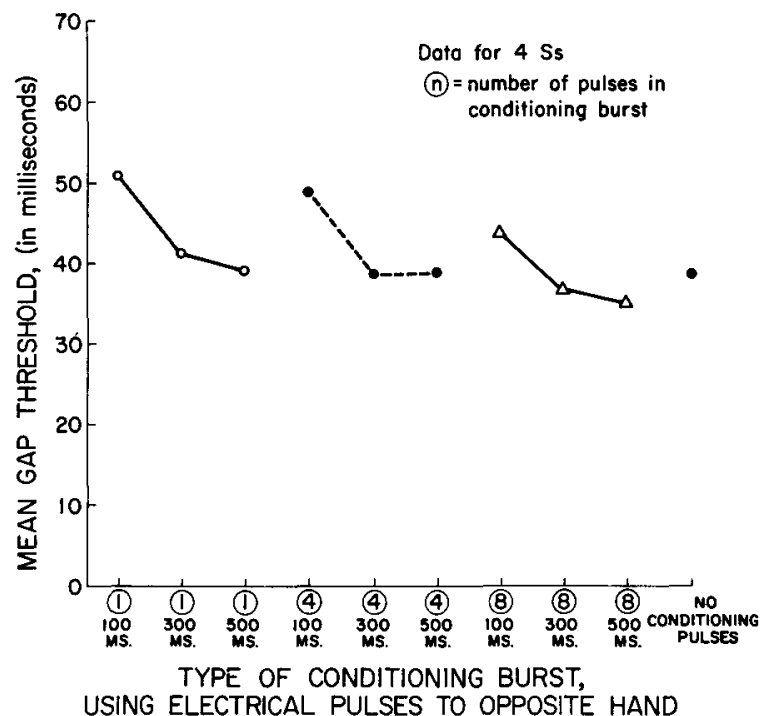

Fig. 4. The data for the experimental trials in which the conditioning burst is made up of a train of electrical stimuli applied to the opposite hand. An elevation of the threshold is observable at the shortest interval between the conditioning and test bursts. The arrangement of the data is the same as for Fig. 2.

the dentist's office, efforts to reproduce this phenomenon in the laboratory have proven the phenomenon to be most elusive.

There appear, then, to be two major sources of difficulty in sensory interaction studies. The first arises from indirect artifacts which modulate what must essentially be considered to be stimulus dimensions. Artifacts of this sort are relatively straightforward and when observed can be adequately controlled. On the other hand, the effects on such complex processes as the perception of pain or the S's estimates of their own performances are not as easily controlled. It was interesting to note that most of our Ss reported that their decisions were harder to make and that they felt as if they were performing less sensitively even in those situations in which little difference in threshold could be detected by our methods. Similarly, the distraction effects of audio analgesia seem to be beyond experimental demonstration with our current conceptual models of psychophysical experiments. These higher mental processes, as such, represent a class of psychological behavior which we in general do not have any satisfactory means of examining. Now let us consider specifically the problem of somatosensory inhibitory interaction.

The gap test which we used in this experiment as a probe to test for both hetero- and homo-modal interaction effects is particularly appropriate since it is exquisitely sensitive to conditioning stimuli. Figure 6 (from Uttal \& Krissoff, 1967) shows the masking effect produced by a preceding stimulus burst applied to the same electrode as the gap test lasts for over half a second and raises the threshold by $190 \%$ in

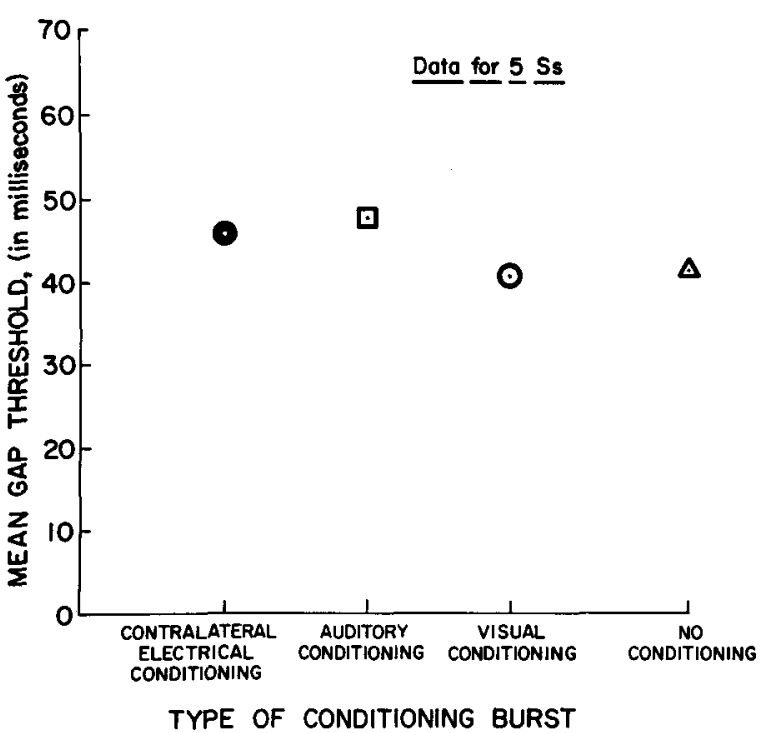

Fig. 5. The data for the experimental trials in which the conditioning burst was $200 \mathrm{msec}$ long and applied at the same time as the test gap. A slight elevation of the threshold is observable for the contralateral electrical conditioning and for auditory conditioning.

some cases. Yet in spite of this very fine ipsilateral sensitivity, the effects in the present experiment for contralateral and heteromodal stimuli demonstrated are relatively small.

The elusiveness of the phenomenon in the laboratory, however, is quite different than that of the

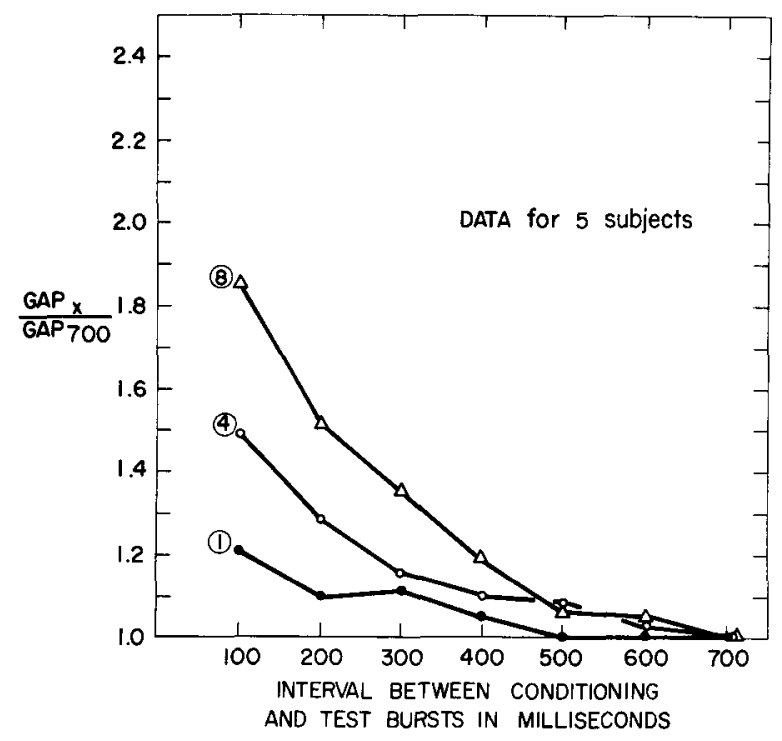

Fig. 6. The data for the trials in which the conditioning stimulus was a burst of electrical pulses applied to the same electrodes as the gap test. This figure is from an earlier paper (Uttal \& Krissoff, 1967) and is presented to provide a reference against which to compare the results of the present experiment. Note that in this figure, data has been normalized by dividing all values by the mean size of the gap for the largest interval used. 
clinical situation. Bender (1952) reports numerous cases in which hemiplegic patients did show significant amounts of "extinction" when opposite sides of the body were simultaneously stimulated by touch or with a pin. This effect may also be an amplitude masking rather than a disruption of the temporal judgment we believe we are studying here. Even in this report, however, the effect was very labile and did not exhibit the stability that might be expected of a result of some very specific lesion of the nerrous system. Bender's comments are always mitigated by the fact that his sample of patients displayed many different kinds of organic or functional disorders which could give rise to the reported effects even in the absence of specific sensory deficits.

There is a subtle distinction which must be made between those studies directed at defining the limits of information processing in the sensory systems and those which reflect the complex manner in which the available information is processed. Factors such as attentiveness, distraction, and misinterpretation of available information are, in a sense, artifacts of our experiment although they represent a perfectly valid set of problems in a different context. As we shall discuss below, it may be possible to actually assign the two different sets of activities to different brain areas.

When we turn to the other senses for data on heteromodal masking, the situation also seems to be that the effects reported are very small. Even in a very well designed experimental situation obtaining positive results (Maier, Bevan, \& Behar, 1961), the effects of auditory stimuli on the flicker fusion frequency results in changes only on the order of $5 \%$, though occasionally even these data were statistically insignificant. A paper by Novak (1965), on the other hand, reports some substantial effects of electrocutaneous conditioning stimuli on visual thresholds for a two-flash acuity measure.

We must also consider the very large number of studies carried out by Russian investigators and summarized by London (1954) in which visual and auditory, as well as a few instances of gustatory, olfactory, and vestibular interaction were demonstrated. London specifically states that the general level of experimental design and control is low. It is, however, interesting to note that in the 506 references of that document there are no references to heteromodal interaction effects on any sort of somatosensory sensitivity.

Though we cannot, on the basis of the present experiment, conclusively resolve the general issue of heteromodal masking, we can discern the general picture for somatosensation. There is a considerable amount of ipsilateral masking obtained but a much smaller amount of contralateral and heteromodal masking, if it exists at all.

These data suggest that the results of the previous experiment on ipsilateral masking were not due to the distraction or attention variables of which we have spoken. Those data, therefore, do represent a good measure of a limit on the information-carrying capacity of the somatosensory system, rather than a reduction in the central utilization of available information.

This, then, brings us to the neurophysiological data on sensory interaction.

Though the data is not completely consistent for the several studies of polysensory interaction on lower animals using compound evoked potentials, it does appear that the interaction effects produced by stimulation with two different stimulus modalities are very much more persistent (up to $1 \mathrm{sec}$ ) in association cortex than in the primary receiving areas (fully recovered in $200 \mathrm{msec}$ ). Both Thompson, Smith, and Bliss (1963) and Dubner and Rutledge (1964) agree on this point though their respective results differ concerning the issue of whether or not the various association areas are differentially sensitive to all incoming stimuli. Thompson and his colleagues, for example, demonstrate that there is a considerable and equivalent inhibitory effect on a second stimulus of any modality in the association cortex when it is preceded by a stimulus of any other modality. The magnitude and duration of the effect is the same regardless of which modality occurs first. They also present data showing a much more moderate persistence of interaction as a function of homomodal interaction in any of the primary sensory areas, but presumably were not able to detect any heteromodal interaction in the primary areas. Mountcastle's data (1957), showing independence of the different submodalities of somatosensation within the primary cortex, also supports this notion.

In sum: The general form of our psychophysical data, no heteromodal interaction for visual stimuli, a small amount of auditory and contralateral homomodal interaction, and a very strong ipsilateral interaction are more in agreement with the pattern of activity of the primary sensory cortex than that of the association cortex. The fact that there is a substantial amount of heteromodal interaction in the association cortex, but none observed psychophysically, suggests that the association cortices do not play a major role in the representation of the basic process involved in the temporal judgment of the gap test. The great discrepancy between the time durations of ipsilateral homomodal stimulation as reported psychophysically (see Fig. 6) and that recorded from the primary cortices suggests that there are other effects than cortical interaction contributing to this interaction. We suggested in the previous paper that it is the after discharge of second order spinal neurons actually flling in the gaps which may account for the greater persistence. The present data add substance to that suggestion. 
It may also be suggested that the small amount of contralateral interaction observed is a result of convergence in the second somatosensory area which is known to receive inputs from both sides of the body but which is not known to receive inputs from visual or acoustic stimulation.

\section{References}

Bender, M. B. Disorders in perception. Springfield, nl.: Charles C. Thomas, 1952.

Bliss, J. C., Crane, H. D., Link, S. W., \& Townsend, J. T. Tactile perception of sequentially presented spatial patterns. Percept. \& Psychophys.. 1966, 1, 125-130.

Dubner, R., \& Rutledge, L. T. Recording and analysis of converging input in cat association cortex. J. Neurophysiol., 1964, $27,620-634$.

Gardner, W. J., \& Licklider, J. C. R. Auditory analgesia in dental operations. J. Amer. Dental Ass., 1959, 59, 1144-1149.

London, I. D. Research on sensory interaction in the Soviet Union. Psychol. Bull, 1954, 51, 531-568.

Maier, Barbara, Bevan, W., \& Behar, I. The effect of auditory stimulation upon the critical flicker frequency for different regions of the visual spectrum. Amer. J. Psychol., 1961, 74, 67-73.

Mountcastle, V. B. Modality and topographic properties of single neurons of cats' somatic sensory cortex. J. Neurophysiol.. 1957, $20,408-434$.

Novak, $\mathbf{S}$. The effect of electrocutaneous digital stimulation on the detection of single and double flashes of light. Psychol. Monogr., 1965,79 , No. 15.
Ogilvie, J. C. Effect of auditory flutter on the visual critical flicker frequency. Canad. J. Psychol., 1956a, 10, 61-68.

Ogilvie, J. C. The interaction of auditory flutter and CFF: The effect of brightness. Canad. J. Psychol., 1956b, 10, 207-210.

Thompson, R. F., Smith, H. E., \& Bliss, D. Auditory, somatic sensory, and visual response interactions and interrelations in association and primary cortical fields of the cat. $J$. Neurophysiol. 1963, 26, 365-378.

Uttal, W. R., \& Krissoff, Madelon. Effect of stimulus pattern on temporal acuity in the somatosensory system. J. exp. Psychol., 1966, 71, 878-883.

Uttal, W. R., \& Krissoff, Madelon. On the refractoriness of somesthetic temporal acuity. Percept. \& Psychophys., 1967, 2, 115-118.

Walker, E. L., \& Sawyer, T. M., Jr. The interaction between flicker frequency and acoustic stimulation. Psychol. Rec., 1961, 11 , 187-191.

\section{Note}

1. The research reported in this paper was supported in part by NIH Grant $08786-03$ and in part by NIH Grant GB 2000 .

2. We would also at this time like to correct an oversight on our part that occurred in an earlier paper in this series. In our earlier paper (Uttal \& Krissoff, 1967) we neglected to cite some of the earlier work by Bliss and his colleagues (1966) on an essentially analogous problem. He had shown that spatial patterns, as well as the temporal patterns we worked with, displayed a similar sort of inhibitory interaction. The fact that his paper also appeared in Percept. \& Psychophys. only compounds our oversight.

(Accepted for publication April 11, 1967.) 